Onkologe $2022 \cdot 28: 180-182$

https://doi.org/10.1007/s00761-021-01094-4

Angenommen: 23. Dezember 2021

(C) The Author(s), under exclusive licence to Springer Medizin Verlag GmbH, ein Teil von Springer Nature 2022

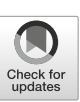

\section{Autor}
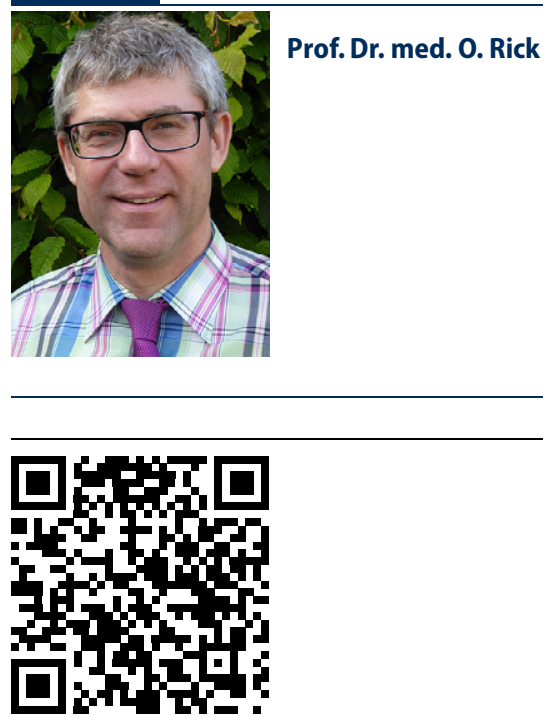

QR-Code scannen \&Beitrag online lesen

\title{
Onkologische Rehabilitation - Teil einer modernen Krebstherapie
}

\author{
O. Rick' ·. Körber ${ }^{2} \cdot$ B. van Oorschot ${ }^{3} \cdot$ K. Höffken ${ }^{4}$ \\ ${ }^{1}$ Klinik Reinhardshöhe, Dr. Ebel Fachkliniken., Bad Wildungen, Deutschland \\ ${ }^{2}$ Klinik Nahetal, Fachklinik für onkologische Rehabilitation und Anschlussrehabilitation (AHB), Bad \\ Kreuznach, Deutschland \\ ${ }^{3}$ Interdisziplinäres Zentrum Palliativmedizin, Universitätsklinikum Würzburg, Würzburg, Deutschland \\ ${ }^{4}$ Berlin, Deutschland
}

Aufgrund der zahlreichen Veränderungen in der akutmedizinischen Behandlung von Patient*innen mit Krebs, insbesondere im Hinblick auf die medikamentösen Therapieverfahren, hat sich nicht nur das Spektrum der akuten Nebenwirkungen, sondern auch das der langfristigen und späten Folgen deutlich verändert. In diesem Kontext unterlag auch die onkologische Rehabilitation in den letzten Jahren einer erheblichen Wandlung und hat sich den neuen Herausforderungen gestellt. Sinn und Zweck der onkologischen Rehabilitation ist, im Rahmen eines zeitlich sehr begrenzten stationären oder ambulanten Aufenthalts die Bedürfnisse der Patient*innen zu erfassen und ein zumeist langfristiges Therapiekonzept zu entwickeln. Dies soll den Patient*innen ermöglichen, über die onkologische Rehabilitation hinaus eine Verbesserung ihrer Langzeit- und Spätfolgen zu erfahren. Mit diesem Ansatz bettet sich die onkologische Rehabilitation hervorragend in das Konzept von Cancer Survivorship ein und stellt damit ein herausragendes Element dar.

\section{I) Die onkologische Rehabilitation bettet sich hervorragend in das Konzept von Cancer Survivorship ein}

Im vorliegenden Leitthema wird auf ausgewählte Langzeit- und Spätfolgen sowie auf besondere Aspekte der onkologischen Rehabilitation eingegangen. Die Schriftleitung hat insbesondere Themen aufgegriffen, die nicht nur im Rahmen der onkologischen Rehabilitation Belang haben, sondern auch in der operativen oder medikamentösen Onkologie wie auch in der Tumornachsorge von den Patient*innen thematisiert werden. Damit soll der interdisziplinäre Brückenschlag gelingen und die ganzheitliche Behandlung von Patient*innen mit Krebs sichtbar werden. Unter diesem Gesichtspunkt widmen sich Güsgen et al. der postoperativen Belastbarkeit und Stabilität von Narben nach viszeralchirurgischen Eingriffen. Bis in die Gegenwart hinein wird Patient*innen mit Krebs nach viszeralchirurgischen Eingriffen nahezu reflexartig eine körperliche Schonung und Entlastung der Bauchdecke für 3 Monate oder mehr empfohlen. Mit genannter Arbeit bringen die Autoren Licht ins Dunkel und stellen den Sachverhalt anhand einer systematischen Literaturrecherche unter Hinzunahme eigener Daten eindrücklich dar.

Nahtlos schließt sich an dieses Kapitel der Beitrag von Aghaijaanpour et al. an. Die verschiedenen thoraxchirurgischen Eingriffe bei Patient*innen mit Krebs werden ausführlich beleuchtet und der Bogen zur Rehabilitation und körperlichen Belastbarkeit geschlagen. Spezifisch wird dabei auch auf die Prähabilitation eingegangen und die Wichtigkeit für ein gutes operatives Outcome dargestellt.

Die Anlage eines Uro- oder Kolostomas stellt nicht nur eine wesentliche Veränderung des Körperbilds dar, sondern hat oftmals dauerhafte Auswirkungen auf das weitere Leben der Patient*innen. Körber u. Hoffmann haben sich aus ihrer 
Hier steht eine Anzeige.

黑 Springer 
langjährigen Erfahrung heraus diesem Thema gewidmet und die erhebliche Bedürftigkeit dieser Patient*innengruppe adressiert. Sie gehen auf die besonderen Aspekte der Langzeitbetreuung der Patient*innen ein, beschreiben mögliche Komplikationen und stellen so die komplexe Problematik in der Betreuung dieser Patient*innen dar.

Den Besonderheiten der Rehabilitation von Adoleszent*innen und jungen Erwachsenen mit Krebs widmen sich König et al. Diese Patient*innengruppe im Alter von 15 bis 39 Jahren zeichnet sich durch eine besondere Bedürftigkeit aus, die sie von älteren Patient*innen mit Krebs unterscheidet. Aspekte wie Familienplanung, Persönlichkeitsentwicklung und berufliche Karriere stehen hier im besonderen Fokus und müssen in der Langzeitbetreuung und damit auch in der onkologischen Rehabilitation Beachtung finden.

Dagegen widmen sich Schubert et al. dem Thema der älteren Patient*innen mit Krebs in der Rehabilitation. In der klinischen Versorgung dieser Patient*innen herrscht oftmals Unklarheit, ob eher eine geriatrische oder eine onkologische Rehabilitation die richtige Entscheidung ist. Mit der Frage "Wer braucht was?" wird dieses Thema fokussiert eingeleitet und die Entscheidungsfindung kontrovers diskutiert. Insbesondere die Entwicklung geriatrischer Assessments hat uns in den letzten Jahren wichtige Instrumente an die Hand gegeben, um dahingehend die richtige Entscheidung zu treffen.

Zu guter Letzt widmet sich der federführende Schriftleiter dem Thema der kognitiven Dysfunktion als Langzeitfolge einer Krebserkrankung. In den letzten zehn bis 15 Jahren sind zu diesem Thema zahlreiche Studien im Hinblick auf Epidemiologie, Pathophysiologie, Prognose, Risikofaktoren, Diagnostik und Therapiemöglichkeiten erschienen. Als Überlappungssyndrom im Rahmen einer chronischen Fatigue oder einer reaktiven depressiven Störung lässt sich die kognitive Dysfunktion zuweilen nur schwer diagnostisch darstellen oder abgrenzen. Sie tritt allerdings auch als eigenständiges Phänomen auf und wird dann oftmals nicht oder erst spät diagnostiziert. Mit dem Beitrag wird dieser Problematik Rechnung getragen und auf die Bedeutung der kognitiven Funktion in der Betreuung von Krebsüberlebenden hingewiesen.

Der vorliegende Schwerpunkt soll allen Kolleg*innen Einblick in das Themenfeld der onkologischen Rehabilitation geben und ihnen bei der Langzeitbetreuung von Patient*innen mit Krebs helfen.

O. Rick

Für die Schriftleitung

\section{K. Höffken}

Für die Herausgebenden

\section{Korrespondenzadresse}

\section{Prof. Dr. med. O. Rick}

Klinik Reinhardshöhe, Dr. Ebel Fachkliniken. Quellenstr. 8-12, 34537 Bad Wildungen, Deutschland

oliver.rick@klinik-reinhardshoehe.de

Interessenkonflikt. O. Rick, J. Körber, B. van Oorschot und K. Höffken geben an, dass kein Interessenkonflikt besteht.
MED UPDATE SEMINARE 2022

\section{Onko Update 2022}

17. Onkologie-Update-Seminar

21.-22. Januar 2022

Berlin und Livestream

28.-29. Februar 2022

Mainz und Livestream

\section{Wiss. Leitung:}

Prof. Dr. Lothar Kanz, Tübingen Prof. Dr. Carsten Bokemeyer, Hamburg Prof. Dr. Ralf Hofheinz, Mannheim Prof. Dr. Andreas Neubauer, Marburg

Unter der Schirmherrschaft der DGIM Mit Unterstützung der DKG www.onko-update.com

\author{
Hämostase Update 2022 \\ 7. Hämophilie-Update- und \\ Hämostaseologie-Update-Seminar \\ 25.-26. März 2022 \\ Berlin und Livestream \\ Wiss. Leitung: \\ Prof. Dr. Edelgard Lindhoff-Last, Frankfurt \\ PD Dr. Robert Klamroth, Berlin \\ Prof. Dr. Sebastian Schellong, Dresden \\ Dr. Ute Scholz, Leipzig \\ Unter der Schirmherrschaft der \\ DGA, DGIM, DGK, DGP \\ www.haemostase-update.com
}

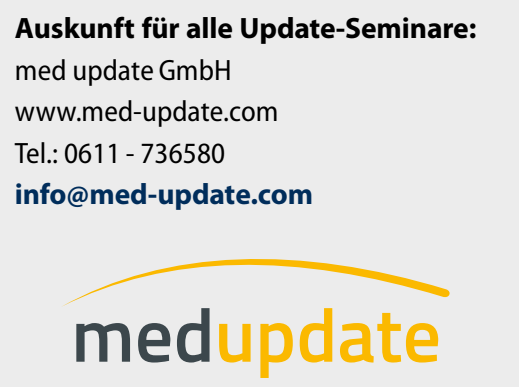

